\title{
Fairness bei Kündigungen des Arbeitsverhältnisses durch Arbeitgeber
}

Die Kündigung des Arbeitsverhältnisses durch den Arbeitgeber beendet einseitig ein auf Dauer angelegtes Rechts- und Sozialverhältnis. Kann Gerechtigkeit in dieser Situation noch eine Rolle spielen? Der Beitrag zeigt auf, dass Gerechtigkeitserwartungen von Arbeitnehmern nicht nur auf den Bestandsschutz ihres Arbeitsverhältnisses gerichtet sind. Vielmehr beurteilen sie auch die Art und Weise, wie eine Kündigung erfolgt, am Maßstab der Gerechtigkeit. Es ist nicht zuletzt das vielfach gescholtene Kündigungsschutzgesetz (KSchG), das die im Arbeitsleben unverzichtbaren Regeln von Gerechtigkeit und Fairness wahrt.

\section{Einleitung}

Erklärt der Arbeitgeber eine Kündigung, so wird hierdurch typischerweise die als dauerhaft erwartete und eingerichtete Erwerbssituation eines Arbeitnehmers gegen seinen Willen beendet. Auch wenn man berücksichtigt, dass die Kündigung des Arbeitsverhältnisses durch den Arbeitgeber mit einem Anteil von rund $32 \%$ an allen Beendigungen von Arbeitsverhältnissen in den Jahren 1999 bis 2001 erst an zweiter Stelle in der Häufigkeitsskala der Arten der Beendigung von Arbeitsverhältnissen steht - nach den Eigenkündigungen der Arbeitnehmer (39 \%) (Pfarr et al. 2005, S. 48) -, werden gegenwärtig in Deutschland im Jahr rund zwei Millionen Kündigungen von Arbeitgebern erklärt (Bielenski/Ullmann 2005, S. 11). Für die meisten Arbeitnehmer ist die ihnen gegenüber erklärte Kündigung ihres Arbeitsverhältnisses eine böse Überraschung. Auch wenn der Schock für die Mehrzahl der sogenannten ordentlichen, d. h. unter Beachtung der Kündigungsfrist erklärten Kündigungen (ca. $85 \%$ ) durch eine gewisse Anpassungszeit gemildert sein mag, bleibt die Wirkung der nicht gewollten Durchkreuzung des Lebens- und Berufsplans. Empirische Untersuchungen zeigen, dass die Akzeptanz einer Kündigung wesentlich davon abhängt, ob die Kündigungsentscheidung des Arbeitgebers und das Kündigungsverfahren als fair wahrgenommen werden (Struck et al. 2006). Die Frage nach der Akzeptanz einer Kündigung des Arbeitsverhältnisses ist ohne Berücksichtigung der moralischen und sozialen Wahrnehmung der Gerechtigkeit des Ergebnisses und der Gerechtigkeit des
Verfahrens nicht angemessen zu beantworten.

In einem Ende 2006 abgeschlossenen rechtsempirischen Forschungsprojekt zur Kündigungspraxis und zum Kündigungsschutz im Arbeitsverhältnis (Höland et al. 2007) haben wir die Bedeutung von Fairness in der Kündigungssituation untersucht. Vor der Darstellung und Diskussion der Daten muss jedoch eine kurze Verständigung über die Begriffe "Gerechtigkeit“ und „Fairness" stehen.

\section{Gerechtigkeit und Fairness - eine begriffliche Vorklärung}

Einen wichtigen Grundstein für die neuere Epoche von Gerechtigkeitstheorien bildet die 1971 erstmalig erschienene „Theory of Justice" von John Rawls (Rawls 1993). Der Grundgedanke dieses Ansatzes kommt bereits in seiner Kennzeichnung zum Ausdruck: Theorie der „Gerechtigkeit als Fairness". Diese (gegen das utilitaristische Konzept des Gesamtnutzens gesetzte) Theorie verknüpft den materiellen Gehalt von Gerechtigkeit mit der Fiktion eines fairen Verfahrens der Verständigung über die Grundsätze, Gerechtigkeit herbeizuführen. In der Tradition eines gesellschaftsvertraglich gefassten Urzustandes, wie ihn unter anderem auch Locke, Rousseau oder Kant gedacht haben, legt Rawls seinem Gerechtigkeitsmodell diejenigen Grundsätze zugrunde, auf die sich freie, vernünftige und gleiche Menschen in einer - nur theoretisch zu denkenden - ursprünglichen Übereinkunft verständigt hätten (Rawls 1993, S. 18). Zu der theoretisch gedachten
Ur-Vertragssituation gehört die Festlegung der Grundsätze der Gerechtigkeit „hinter einem Schleier des Nichtwissens" (Rawls 1993, S. 29). Niemand kennt seine Stellung in der Gesellschaft, seine Klasse oder seinen Status, niemand weiß, über welche natürlichen Gaben wie Intelligenz oder Körperkraft er verfügen können wird.

Für die Anwendbarkeit der Gerechtigkeitstheorie von John Rawls auf Forschungsdaten im Zusammenhang mit Kündigungsstreitigkeiten wird ein weiterer Baustein im Rawls'schen Theoriengebäude von Bedeutung sein: die Institution. Institutionen erwachsen aus den urvertraglich vereinbarten Grundsätzen der sozialen Gerechtigkeit und bilden die von diesen Grundsätzen getragene Grundstruktur der Gesellschaft. Unter einer Institution versteht Rawls ,ein öffentliches Regelsystem, das Ämter und Positionen bestimmt mit ihren Rechten und Pflichten, Machtbefugnissen und Schutzzonen u. ä." (Rawls 1993, S. 74). Beispiele sind Spiele, Riten, Gerichtsverfahren, Parlamente, Märkte, Eigentumssysteme.

Weitere wichtige Zuflüsse zur neueren Gerechtigkeitsdiskussion sind rechtssoziologische und rechts- wie sozialpsychologische Erkenntnisse zur Verfahrensgerechtigkeit. Sie haben wesentliche Anregungen aus

Armin Höland, Prof. Dr., Universität Halle-
Wittenberg. Arbeitsschwerpunkte: Bürger-
liches Recht, Arbeitsrecht, Recht der sozialen
Sicherung, Rechtssoziologie.
e-mail: Hoeland@jura.uni-halle.de
Nadine Zeibig, Wissenschaftlerin im Projekt
Regulierung des Arbeitsmarkts (REGAM) im
WSI in der Hans-Böckler-Stiftung.
e-mail: nadine-zeibig@boeckler.de


dem Ende der 1960er Jahre in den USA eröffneten Themenfeld der „Procedural justice" bezogen, insbesondere aus den Studien von Thibaut und Walker (1975) sowie von Lind und Tyler (Lind/Tyler 1988; Tyler 1990, 2005), die sich ihrerseits auf die Gerechtigkeitsanalysen von Leventhal (Leventhal 1980) bezogen. In der deutschen Rechtssoziologie sind diese Forschungsund Theorieansätze vor allem durch Klaus F. Röhl (1987, S. 157ff.; 1993; 1997), Günter Bierbrauer (1995) und Stefan Machura $(2000 ; 2003 ; 2006)$ aufgenommen und weiterentwickelt worden. Bei aller Vielfalt der Deutung der Ursachen ist den Ansätzen der Verfahrensgerechtigkeit die auch empirisch belegte Erkenntnis gemeinsam, dass die Fairness von Verfahren wesentlich dazu beiträgt, Ergebnisse auch dann akzeptabel zu machen, wenn sie hinter dem erwarteten Nutzen zurückbleiben bzw. ungünstig oder auch belastend ausfallen. Der Effekt der Abmilderung der Folgen durch das Verfahren gilt selbst für die Opfer von Straftaten im Hinblick auf ihre Wahrnehmung der Fairness in den polizeilichen und staatsanwaltschaftlichen Ermittlungsverfahren (Wemmers 1996).

In der Weiterentwicklung des Konzepts der Verfahrensgerechtigkeit durch Tyler und Lind kommt die subjektive Voraussetzung des Gerechtigkeitsempfindens in einer anderen Fassung zum Ausdruck. Dabei ist der Blickwinkel von der Kontrolle über die Abläufe und Ergebnisse des Verfahrens verlagert worden zu einem Beziehungsmodell der Verfahrensgerechtigkeit. Es beruht auf der Annahme, dass Verfahren als fair wahrgenommen werden, wenn sie geeignet sind, die grundlegenden Werte einer sozialen Gruppe - wie beispielsweise der Arbeitnehmer - und der dieser Gruppe angehörenden Individuen zur Geltung zu bringen. Unter Einbeziehung dieser Überlegungen sehen Tyler und Lind die Fairness von Verfahren durch die drei Merkmale „standing“" (die Wertschätzung der Verfahrensbeteiligten und ihre würdige und respektvolle Behandlung im Verfahren), "trust" (Vertrauen in die ethische Grundhaltung, soziale Aufmerksamkeit und Verständnis der das Verfahren gestaltenden Personen) und „neutrality“ (Redlichkeit und Genauigkeit sowie Unvoreingenommenheit der Akteure im Vollzug des Verfahrens) bestimmt (Wemmers 1996, S. 117ff.; Machura 2000, S. 11).

Einen anderen Zugang zu Verfahren hat die einflussreiche, 1969 erstmalig ver- öffentlichte soziologische Analyse der „Legitimation durch Verfahren“"von Niklas Luhmann eröffnet (Luhmann 1983; vgl. auch die ausführliche Besprechung der Luhmannschen Analyse durch Machura 1993). Die Überlegungen von Luhmann nehmen ihren Ausgangspunkt in dem das Verfahren, insbesondere das Gerichtsverfahren, bestimmenden Verhältnis zwischen Wahrheitswert und Entscheidungsnotwendigkeit. Voraussetzung für die Legitimation von Entscheidungen ist ein effektives, möglichst störungsfreies Lernen im sozialen System (Luhmann 1983, S. 35). Mit dieser Deutung der Legitimation von Entscheidungen als institutionalisierter Lernprozess mündet der Ansatz von Luhmann darin, dass er die durch Verfahren gewonnene Legitimation beschreibt als eine Umstrukturierung des Erwartens durch den faktischen Kommunikationsprozess, der nach Maßgabe rechtlicher Regelungen abläuft (Luhmann 1983, S. 37).

Unter einem anderen Blickwinkel sind die spieltheoretischen und spielempirischen Erkenntnisse der Verfahrensökonomie zur Gerechtigkeit und Fairness gewonnen (Magen 2005a; Alewell/Nicklisch 2006). Sie führen einen Gesichtspunkt in die Gerechtigkeitsanalyse ein, der auch im Hinblick auf die Kündigung eines Arbeitsverhältnisses Beachtung verdient: die das Verhalten steuernde Wirkung der Reziprozität. Die Erklärungskraft dieser anthropologischen Konstante ist so groß, dass sich Fairnesstheorien nach dem Vorschlag von Stefan Magen in die beiden Gruppen der Theorien der Verteilungsgerechtigkeit einerseits und der Theorien der Reziprozität andererseits einteilen lassen (Magen 2005a, S. 33). Im Unterschied zur Betrachtung des gerechten Ergebnisses einer Distribution machen die Theorien der Reziprozität darauf aufmerksam, dass faire und freundliche Absichten der Mitspieler auf die Bewertung von Ergebnissen der Verteilung Einfluss nehmen (Magen 2005a, S. 36). Mit diesem Ansatz rücken sie die Art und Weise des Zustandekommens der Ergebnisse in den Blick und stellen damit eine Verbindung zur Verfahrensdimension von Gerechtigkeit her. Im Hinblick auf die Art und Weise des Zustandekommens von Ergebnissen lassen sich im Spielexperiment Fairnesspräferenzen der Spieler modellieren (Magen 2005a, S. 36ff.). Auf diese Weise ist es möglich, die beiden grundsätzlichen Ausprägungen der Gerechtigkeit, die Gerechtigkeit der Verteilung und des Verfahrens, in ihrem Verhältnis zueinander experimentell nachzubilden.

\section{Fairness im Zusammen- hang mit der Kündigung des Arbeitsverhältnisses durch den Arbeitgeber}

Arbeitsverträge sind als implizite oder psychologische Verträge (Sadowski 2002, S. 72ff.; Durkheim 1986, S. 189ff.; Fehr et al. 2004) in besonderem Maße abhängig von der sie begleitenden und über ihren ausdrücklichen schuldrechtlichen Verpflichtungsgehalt hinausreichenden Erwartungssicherheit. Nicht nur in spieltheoretisch nachgestellten Situationen (Magen 2005b; ders. 2005a, S. 33, 36ff.), sondern auch in der Lebenswirklichkeit der Arbeitswelt kommt die Wirkung der Reziprozität als eine den Austausch sichernde und das längerfristige Engagement der Beteiligten motivierende Bedingung zum Tragen. Gegen die Reziprozitätserwartung der Arbeitsvertragsparteien setzt die Kündigung des Arbeitsverhältnisses durch den Arbeitgeber das Signal zum Abbruch der Arbeitsbeziehung. Im Grundsatz gilt das auch umgekehrt für die Kündigung durch den Arbeitnehmer, typischerweise sind die Wirkungen jedoch weniger belastend, jedenfalls weniger persönlich belastend. Die auf Umfragen beruhenden Ergebnisse des Forschungsprojektes "Arbeit und Fairness"1 können für die Bundesrepublik Deutschland wie auch im Vergleich mit den USA und Kanada deutliche Unterschiede aufzeigen, wann eine Kündigung als ungerecht wahrgenommen wird. Die Unterschiede folgen im Grunde dem Muster der Reziprozität. Hat der Arbeitgeber sich um die Vermeidung der Kündigung(en) bemüht, wird das Ergebnis signifikant seltener als ungerecht wahrgenommen, als wenn solche Bemühungen unterlassen worden sind oder wenn gar das mit Personalreduzie-

\footnotetext{
Das im Jahr 2004 im Verbund der Universitäten Jena und Hannover durchgeführte Projekt "Arbeit und Fairness" hat aus der telefonischen Befragung von über 3.000 Personen empirische Erkenntnisse zur Wahrnehmung von Gerechtigkeit bei der Beendigung von Arbeitsverhältnissen gewinnen können (Struck et al. 2006); vgl. dazu auch den Beitrag von Krause in diesem Heft.
} 
rung beauftragte Management Gratifikationen erhält (Stephan 2006, S. 3; Gerlach et al. 2006, S. 17f.; Krause et al. 2006, S. 35ff.). Ebenfalls abgemildert wird der Eindruck der Ungerechtigkeit durch die Bereitschaft des Unternehmens, Abfindungen zu zahlen oder Hilfestellung bei der Stellensuche zu geben.

Die Untersuchungen aus dem Projekt „Arbeit und Fairness" können eine weitere interessante Wirkung feststellen. Allein mit der Existenz des Kündigungsschutzgesetzes verknüpfen sich Erwartungen der Gerechtigkeit und Fairness (Krause 2006a, S. 105ff.). Das Kündigungsschutzgesetz wirkt wie ein staatliches Fairnessversprechen, auch wenn es im Konfliktfall erst aktiviert werden muss. Dieser Befund lässt sich an die Figur der Institution in der Gerechtigkeitstheorie von John Rawls anschließen. Das Rechtssystem des Kündigungsschutzes ist in diesem Sinne ein öffentliches Regelsystem, das die auf die abhängige Arbeit bezogenen Grundsätze der Gerechtigkeit als allgemeine Werteordnung und im konkreten Bezug auf Konfliktfälle zur Anwendung bringt. Bereits die bloße Existenz der Institution „Kündigungsschutz" hat einen wesentlichen Anteil an der allgemeinen Wahrnehmung einer Arbeitsgesellschaft als gerecht und fair (Krause 2006b).

Auch das in den Jahren 2003 bis 2006 an der Juristischen Fakultät der Martin-Luther-Universität Halle-Wittenberg durchgeführte arbeitsrechtssoziologische Forschungsprojekt zur Kündigungspraxis und zum Kündigungsschutz im Arbeitsverhältnis aus Sicht des arbeitsgerichtlichen Verfahrens ${ }^{2}$ liefert - wenn auch beschränkt auf die vor die Arbeitsgerichtsbarkeit gebrachten Kündigungsstreitigkeiten ${ }^{3}$ - empirische Belege für die Bedeutung der Fairness in den betrieblichen und gerichtlichen Verfahren und für die Akzeptanz der Entscheidungen. Diese Befunde werden im Folgenden vorgestellt.

\section{Die Anhörung der Arbeit- nehmer vor Kündigungen}

Die Verfahrensgerechtigkeit (procedural justice) bietet einen Maßstab dafür, ob die Art und Weise, wie ein Ergebnis zustande kommt, als fair und gerecht beurteilt wird. Bezogen auf die Situation der Kündigung eines Arbeitsverhältnisses stellt sich die Frage, inwieweit z. B. die von der Kündigung betroffenen Arbeitnehmer respektvoll behandelt und ihre Bedenken und Bewertungen durch vorherige Anhörung in die Kündigungsentscheidung einbezogen werden. Sucht der Arbeitgeber vor einer Kündigung das Gespräch mit dem Arbeitnehmer, so kann das dazu beitragen, dass Letzterer die Entscheidung als gerecht akzeptiert. Auf die Bedeutung der Anhörung des Arbeitnehmers vor der Kündigung weisen auch die Erkenntnisse des Forschungsprojektes „Arbeit und Fairness“ sowie die Untersuchungen von Christian Pfeifer hin. Danach werden Kündigungen von betroffenen Arbeitnehmern, aber auch von unbeteiligten Dritten, deutlich häufiger als gerecht bewertet, wenn die von einer Kündigung Betroffenen in den Entscheidungsprozess einer Kündigung frühzeitig einbezogen werden (Stephan et al. 2006, S. 15; Krause et al. 2006, S. 62; Pfeifer 2004, S. 141). Betriebliche Daten, ob der Arbeitgeber den Arbeitnehmer vor Ausspruch der Kündigung angehört hat, gibt es nicht. Jedoch liefern die Daten des Forschungsprojektes der Martin-Luther-Universität (vgl. Fn. 2) einen Anhaltspunkt zur Häufigkeit von Anhörungen vor Kündigungserklärungen, zumindest für die vor die Arbeitsgerichte gelangten Kündigungen. Hiernach hat der Arbeitgeber den Arbeitnehmer nur in $19 \%$ der Kündigungsfälle, die vor die Arbeits- und Landesarbeitsgerichte gelangt sind, vor der Kündigung angehört, in $56 \%$ nicht (in $25 \%$ der Fälle war dies nicht feststellbar). Allerdings waren $57 \%$ der gerichtlich verhandelten Fälle betriebsbedingte Kündigungen. Vor solchen Kündigungen mag eine Anhörung des Arbeitnehmers weniger notwendig erscheinen, da der Grund für die Kündigung vom Arbeitnehmer ohnehin nicht beeinflusst werden kann. Zwingend erforderlich ist die vorherige Anhörung nach der Rechtsprechung des Bundesarbeitsgerichts nur in Fällen der sogenannten Verdachtskündigung. Eine schuldhaft unterlassene Anhörung führt zur Unwirksamkeit der Verdachtskündigung (Müller-Glöge 2006, $\$ 626$, Rn. 213). Gerade deswegen ist es auffällig, dass in nur $58 \%$ der vor die Arbeits- und Landesarbeitsgerichte gelangten und den Arbeitnehmern gegenüber begründeten Verdachtskündigungen der Arbeitnehmer angehört wurde. Eine Erklärung dafür könnte in der Unkenntnis des Arbeitgebers von dem Wirksamkeitserfordernis der An- hörung liegen, aber auch die wissentliche Missachtung aus Gleichgültigkeit oder die Hoffnung, der Arbeitnehmer werde keine Klage erheben, könnten eine Erklärung sein.

$\mathrm{Ob}$ eine Entscheidung als gerecht wahrgenommen wird, hängt weiterhin davon ab, dass sie begründet wird. Die Begründung einer Kündigung durch den Arbeitgeber ist ein Zeichen für respektvollen Umgang mit dem Arbeitnehmer und damit ein Element des psychologischen Vertrages. Arbeitnehmer akzeptieren ein negatives Ergebnis eher, wenn dieses plausibel begründet wird (zu diesem Aspekt ,informatorischer Gerechtigkeit" siehe Brockner et al. 1994, S. 406; 1990). Das deutsche Kündigungsschutzrecht verlangt für die Kündigung des Arbeitsverhältnisses durch den Arbeitgeber zwar einen diese rechtfertigenden Grund, aber nicht notwendigerweise eine Begründung. Eine gesetzliche Ausnahme bildet die außerordentliche Kündigung. Hier hat der Arbeitgeber dem Arbeitnehmer auf dessen Verlangen nach $\$ 626$ Abs. 2 S. 3 BGB die Gründe für die Kündigung mitzuteilen. Anhaltspunkte dafür, dass die Begründung von Kündigungen im Arbeitsleben gleichwohl generell verbreitet ist, liefern unsere Projektdaten: Danach haben Arbeitgeber in $66 \%$ der vor die Arbeits- und Landesarbeitsgerichte gelangten Kündigungen Begründungen mitgeteilt, in zwei Drittel dieser Fälle bereits im Kündigungsschreiben. In $25 \%$ wurde die Kündigung nicht begründet, in $9 \%$ der Fälle war dies nicht feststellbar.

\footnotetext{
2 Im Rahmen des von der Hans-Böckler-Stiftung finanzierten Forschungsprojekts zu Kündigungspraxis und Kündigungsschutz im Arbeitsverhältnis aus Sicht des arbeitsgerichtlichen Verfahrens wurden alle 1.119 Vorsitzenden Richterinnen und Richter der Arbeits- und Landesarbeitsgerichte in Deutschland postalisch befragt und um die Auswertung nach Erledigungsarten differenzierter Kündigungsklageverfahren (ohne öffentlichen Dienst) gebeten. Nähere Informationen zum Projekt und zu den Ergebnissen dieser Studie siehe Höland et al. 2007.

3 Rund $15 \%$ der Gekündigten erheben Klage vor dem Arbeitsgericht gegen die vom Arbeitgeber erklärte Kündigung (Pfarr et al. 2005, S. 58).
} 


\section{Die Anhörung des Betriebsrats vor Kündigungen}

Die nach $₫ 102$ Abs. 1 BetrVG zwingend vorgeschriebene Anhörung des Betriebsrats vor Kündigungen des Arbeitgebers ist für die Wahrnehmung von Gerechtigkeit von großer Bedeutung. Die Stellungnahme des Betriebsrates kann den Arbeitgeber von Kündigungsvorhaben abbringen oder ihn bei geplanten Betriebsänderungen zum Abschluss eines Interessenausgleichs und Sozialplans veranlassen. Die Beteiligung des Betriebsrates an Kündigungsverfahren kann Informationsasymmetrien verhindern und in gewisser Weise auch das Eigeninteresse des Arbeitgebers begrenzen (Pfeifer 2004, S. 136). Werden Arbeitnehmervertretungen an Kündigungsentscheidungen beteiligt, so gelten die Kündigungen als wesentlich gerechter (Pfeifer 2004, S. 137; Brockner et al. 2004, S. 96).

Daten einer in den Jahren 1999-2001 vom Wirtschafts- und Sozialwissenschaftlichen Institut in der Hans-Böckler-Stiftung durchgeführten Untersuchung zeigen, das bei $57 \%$ der Gekündigten aus Betrieben mit Betriebsrat in der Privatwirtschaft dieser vor Ausspruch der Arbeitgeberkündigung informiert wurde. ${ }^{4} 12 \%$ der Gekündigten gaben an, dass der Betriebsrat nicht informiert wurde, $31 \%$ konnten die Frage nach einer erfolgten Betriebsratsinformation nicht beantworten. Nach den Daten des Forschungsprojekts der Martin-Luther-Universität wurde der Betriebsrat vor Kündigungen, die vor die Arbeits- und Landesarbeitsgerichte gelangten, in $91 \%$ (ArbG) bzw. $94 \%$ (LAG) angehört, in $2 \%$ (ArbG) bzw. 0 \% (LAG) erfolgte die erforderliche Betriebsratsanhörung nicht (Höland et al. 2007, S. 100). Die Betriebsräte haben in $26 \%$ der vor die Arbeitsgerichte gelangten Kündigungen (LAG $23 \%$ ) der Kündigung ausdrücklich zugestimmt, in $26 \%$ (LAG $28 \%$ ) der Fälle jedoch der Kündigung ausdrücklich widersprochen. Insgesamt zeigen die Daten, dass Arbeitgeber in der deutlichen Mehrzahl der vor die Arbeits- und Landesarbeitsgerichte gelangten Fälle die vom Gesetz geforderte Betriebsratsanhörung vor Ausspruch einer Kündigung auch tatsächlich durchführen.

Die Beteiligung des Betriebsrates an Kündigungsvorhaben ist auch im Hinblick auf die Klagebereitschaft gekündigter Arbeitnehmer von Bedeutung. So belegen empirische Studien, dass der Widerspruch des Betriebsrates die Klagequote gegen Kündigungen erhöht (Pfarr et al. 2005, S. 62, 64), da Arbeitnehmer im Widerspruch des Betriebsrates häufig ein Indiz fehlender Rechtmäßigkeit der Kündigung sehen und so motiviert werden, den ihnen zustehenden Bestandsschutz geltend zu machen.

\section{Die Sozialauswahl bei betriebsbedingten Kündigungen}

Gemäß $₫ 1$ Abs. 3 KSchG hat der Arbeitgeber vor einer betriebsbedingten Kündigung in der Regel eine Sozialauswahl durchzuführen. Dabei hat er anhand von vier sozialen Auswahlkriterien (Betriebszugehörigkeit, Alter, Unterhaltspflichten und Schwerbehinderung) unter den vergleichbaren Arbeitnehmern diejenigen für die Kündigung auszuwählen, die am wenigsten sozial schutzwürdig sind. Mit dieser Regelung hat der Gesetzgeber im Zusammenhang mit der Kündigung von Arbeitsverhältnissen eine elementare Ausprägung der Verteilungsgerechtigkeit (distributive justice) in das Gesetz aufgenommen. ${ }^{5}$ Verteilungsgerechtigkeit befasst sich damit, inwieweit Ergebnisse einer Entscheidung bzw. die Verteilung von Gütern und Lasten als fair und gerecht wahrgenommen werden. Für die Beurteilung der Verteilung von Gütern, Lasten und Ressourcen als gerecht kommen drei grundlegende Prinzipien in Betracht: das Beitragsprinzip (equity), das Gleichheitsprinzip (equality) und das Bedarfsprinzip (need) (Röhl 1993, S. 7).

Im Zusammenhang mit der vom Arbeitgeber durchzuführenden Sozialauswahl bei betriebsbedingten Kündigungen hat sich der Gesetzgeber in $\$ 1$ Abs. 3 KSchG bei den sozialen Auswahlkriterien durch die vorgegebene Einbeziehung des Alters, der Unterhaltspflichten sowie der Schwerbehinderung vorrangig für das Verteilungsprinzip der Bedürftigkeit entschieden. Die soziale Auswahl im Sinne des $₫ 1$ Abs. 3 KSchG enthält unter dem Gesichtspunkt der Verteilungsgerechtigkeit aber auch Elemente des Beitragsprinzips. So muss der Arbeitgeber die Betriebszugehörigkeitsdauer bei der Auswahl des zu Kündigenden beachten. Langjährige Mit- arbeiter haben viel Zeit und Arbeit in das Unternehmen investiert, langjähriges Vertrauen in die Vertragsbeziehung wurde aufgebaut. Der Schutz der Betriebszugehörigkeit verwirklicht somit Verteilungsgerechtigkeit anhand des Beitragsprinzips.

Nach den Befunden von Höland et al. (2007) wurde bei $41 \%$ der Kündigungsschutzklagen gegen betriebsbedingte Kündigungen eine Sozialauswahl durchgeführt. Bei den Landesarbeitsgerichten wurde in $39 \%$ der Berufungen gegen betriebsbedingte Kündigungen eine Sozialauswahl vorgenommen. In $45 \%$ (ArbG) bzw. $54 \%$ (LAG) der Fälle erfolgte keine Sozialauswahl. Allerdings wäre nur in $18 \%$ (ArbG) bzw. $12 \%$ (LAG) der Fälle, in denen keine Sozialauswahl stattfand, eine solche erforderlich gewesen (ebd., S. 117f.). Nach den Forschungsdaten führen Arbeitgeber demnach in der Mehrzahl der betriebsbedingten Kündigungen die vom Gesetz vorgeschriebene Sozialauswahl durch. In der öffentlichen Debatte um die Wirkungen des arbeitsrechtlichen Kündigungsschutzes in Deutschland wird häufig kritisiert, dass die Anforderungen, die die Rechtsprechung für eine ordnungsgemäße Sozialauswahl verlangt, die Arbeitgeber vor unüberwindbare Hürden stellen würde. Aus Sicht des arbeitsgerichtlichen Kündigungsklageverfahrens kann diese Behauptung nicht bestätigt werden. Nach den Aktenanalysen war die Sozialauswahl in den Verfahren vor den Arbeitsgerichten nur in $22 \%$ der Fälle fehlerhaft, vor den Landesarbeitsgerichten lag der entsprechende Wert bei $19 \%$.

\section{Die gütliche Beilegung des Rechtsstreits durch (Abfindungs-)Vergleich}

Klaus F. Röhl definiert Verfahren als „,institutionelle Prozesse der Entscheidungs- und Konsensbildung“ (Röhl 1993, S. 3). Auch

\footnotetext{
4 Eigene Berechnungen anhand der Daten der WSIBeendigungsbefragung aus den Jahren 19992001. Nähere Informationen zu dieser Studie und weitere Ergebnisse: Pfarr et al. (2005).

5 Auch bei Kündigungen von Arbeitnehmern, die nicht unter das KSchG fallen, hat der Arbeitgeber unter dem Gesichtspunkt der Wahrung von Verfahrens- und Verteilungsgerechtigkeit ein gewisses $\mathrm{Maß}$ an sozialer Rücksichtnahme zu beachten, BVerfGE 97, 169ff. Allgemein hierzu Hakel (2006).
} 
der Abschluss eines Vergleichs vor den Gerichten für Arbeitssachen ist das Ergebnis einer Konsensbildung, resultierend aus regelgebundenen Verhandlungen. Gemäß $\$ 779$ Abs. 1 BGB ist ein Vergleich ein Vertrag, durch den der Streit oder die Ungewissheit der Parteien über ein Rechtsverhältnis im Wege des gegenseitigen Nachgebens beseitigt wird. $65 \%$ der Kündigungsklageverfahren vor den Arbeitsgerichten und $51 \%$ der Berufungsverfahren in Kündigungssachen vor den Landesarbeitsgerichten werden in Deutschland durch gerichtlichen Vergleich erledigt (Höland et al. 2007, S. 147). Nur 11 \% der Kündigungsklageverfahren vor den Arbeitsgerichten (LAG $30 \%$ ) werden durch streitiges Urteil, d. h. durch Entscheidung des Richters, beendet. Der Prozessvergleich ist ein flexibles Mittel der Streitbeilegung. Die gütliche Einigung dient dem Rechtsschutz und der Rechtssicherheit nicht weniger, dem Abbau von Spannungen und der Befriedung von Parteien aber häufig mehr als die richterliche Entscheidung (Rudolph 1983, S. 38). Im Rahmen der Vergleichsgespräche wird den Parteien die Tragweite der inhaltlichen Entscheidung vor Augen geführt, die Interessen werden in einen frei verhandelten und im Regelfall angemessenen Ausgleich gebracht, die Anforderungen an ein gerechtes Verfahren werden erfüllt. Entlassungen werden auch dann deutlich eher akzeptiert und als gerecht empfunden, wenn die Betroffenen einen finanziellen Ausgleich erhalten (Krause et al. 2006, S. 55; Pfeifer 2004, S. 141). Zur Zusage der Zahlung einer Abfindung kommt es in $75 \%$ der Vergleiche in Kündigungsklageverfahren vor den Arbeitsgerichten (LAG $79 \%$ ). Die durchschnittliche Höhe vor Gericht vereinbarter Abfindungszahlungen liegt bei 0,57 Bruttomonatsgehältern pro Beschäftigungsjahr (LAG 0,89) (Höland et al. 2007, S. 160).

Insgesamt werden allerdings nur bei $10 \%$ aller Beendigungen von Arbeitsverhältnissen Abfindungen gezahlt (Pfarr et al. 2005, S. 66). Darin sind Abfindungen aus Sozialplan und Abfindungen nach Klage- erhebung bereits enthalten. In Zeiten anhaltend hoher Arbeitslosigkeit und wiederholter Kürzungen von Lohnersatzleistungen gewinnt die Abfindung besondere Bedeutung als finanzielle Überbrückungshilfe. Ist es unter diesem Gesichtspunkt gerecht, wenn es von einer Klage und dem Verhandlungsgeschick des Arbeitnehmers abhängt, ob eine Abfindung gezahlt wird? Wohl nicht. Ein Beispiel für ein allem Anschein nach gerechteres Abfindungssystem bietet Österreich. Österreich hat im Jahre 2002 sein Abfindungsrecht (Abfertigungsrecht) reformiert (Leuthner et al. 2003). Ein Beweggrund war die ungerechte Verteilung der Abfertigungen. Nur rund $18 \%$ der Arbeitnehmer in Österreich kamen vor der Reform in den Genuss der Abfertigungszahlung. Nach der neuen, seit 2003 in Österreich geltenden Rechtslage zahlt der Arbeitgeber für jeden Arbeitnehmer mit Beginn des zweiten Monats des Arbeitsverhältnisses Beiträge in Höhe von 1,53 \% der monatlichen Bruttovergütung in sogenannte Mitarbeitervorsorgekassen ein. Dadurch erwirbt jeder Arbeitnehmer im Laufe seines Erwerbslebens Ansprüche auf Abfertigung. Dieser Anspruch besteht unabhängig von der Art der Beendigung des Arbeitsverhältnisses.

\section{Zusammenfassung}

Die Kündigung eines Arbeitsverhältnisses durch den Arbeitgeber beendet ein auf Dauer angelegtes Rechts- und Sozialverhältnis, an das sich aus Sicht der Arbeitnehmer im Regelfall gewichtige berufliche und persönliche Erwartungen knüpfen. Werden sie durch die Kündigungsentscheidung des Arbeitgebers enttäuscht, fällt es schwer sich vorzustellen, dass Arbeitnehmer das als fair und gerecht empfinden könnten. Ergebnisse der empirischen Rechts- und Sozialforschung können gleichwohl Belege dafür liefern, dass es auch in der Krisensituation einer Kündi- gung Verfahrens- und Entscheidungsmöglichkeiten gibt, die als mehr oder weniger fair und gerecht wahrgenommen werden. Dafür ist schon das Bestehen des Kündigungsschutzgesetzes bedeutsam. Es institutionalisiert Gerechtigkeitserwartungen von Arbeitnehmern in Bezug auf den Bestandsschutz ihres Arbeitsverhältnisses und gibt den Erwartungen materiell- und verfahrensrechtlichen Ausdruck. Der in Recht gefasste Gedanke des Bestandsschutzes im Arbeitsverhältnis erweist sich damit als ein tragendes Element im Rechtsund Gerechtigkeitsverständnis der industriellen Beziehungen in Deutschland. Forschungsdaten zeigen ferner, dass und wie sich Verfahren und Ergebnisse der Kündigung auf die Wahrnehmung von Fairness und Gerechtigkeit auswirken. Die Anhörung der Arbeitnehmer und des Betriebsrates vor der Kündigung, die Sozialauswahl bei betriebsbedingten Kündigungen und die Gestaltungsmöglichkeiten im Rahmen von Vergleichsgesprächen sind für das Gerechtigkeitsempfinden der Arbeitnehmer relevante Größen. Die Gerechtigkeitswahrnehmung von Kündigungen spielt allerdings nicht nur für die von der Kündigung Betroffenen eine Rolle, sondern wirkt sich auch auf die im Betrieb verbleibende Belegschaft aus. So zeigen empirische Daten, dass Arbeitgeberkündigungen grundsätzlich die Zusammenarbeit zwischen den verbleibenden Beschäftigten und den Vorgesetzten beeinträchtigen (Struck 2006, S. 91). Bemühen sich Arbeitgeber nicht darum, Kündigungen zu vermeiden oder beziehen sie die von der Kündigung betroffenen Arbeitnehmer nicht frühzeitig in die Kündigungsentscheidung ein, so sinken die Identifikation mit dem Unternehmen und das Engagement der verbleibenden Belegschaft deutlich und die Bereitschaft zur Eigenkündigung steigt (Struck 2006, S. 96). All das sollte bedacht werden, wenn über eine Abschaffung bzw. Reduzierung des Kündigungsschutzes nachgedacht wird. 
Alewell, D./Nicklisch, A. (2006): Wage Differentials, Fairness, and Social Comparison: An experimental study of the Co-Employment of Permanent and Temporary Agency Workers, Bonn, http://www.coll.mpg.de/ pdf_dat/2006_8online.pdf

Bielenski, H./Ullmann, K. (2005): Arbeitgeberkündigungen und Klagequote, Bundesarbeitsblatt 10, S. 4-13

Bierbrauer, G. (Hrsg.) (1995): Verfahrensgerechtigkeit. Rechtspsychologische Forschungsbeiträge für die Justizpraxis, Köln Brockner, J./Konovsky, M./Cooper-Schneider, R./Folger, R./Martin, C./Bies, R. J. (1994): Interactive Effects of Procedural Justice and Outcome Negativity on Victims and Survivors of Job Loss, in: Academy of Management Journal, pp. 397-409

Brockner, J./De Witt, R. L. /Grover, S./Reed, T. (1990): When it is especially important to explain why: Factors affecting the relationship between managers' explanations of layoff and survivors' reactions to the layoff, in: Journal of Experimental Social Psychology, pp. 389-407 Brockner, J./Spreitzer, G./Mishra, A./Hochwarter, W./Weinberg, J. (2004): Perceived Control as an Antidote to the Negative Effects of Layoffs on Survivors' Organizational Commitment and Job Performance, in: Administrative Science Quarterly, pp. 76-100

Durkheim, E. (1986): De la division du travail social, Paris

Fehr, E./Klein, A./Schmidt, K. M. (2004): Contracts, fairness, and incentives, München 2004, CESifo working paper no. 1215, Labour Markets Gerlach, K./Levine, D./Stephan, G./Struck, O. (2006): The Acceptability of Layoffs and Pay Cuts: Comparing North America with Germany, IABDiscussionPaper 1

Hakel, C. (2006): Gerechtigkeitsprobleme bei der Beendigung von Arbeitsverhältnissen: ein Rechtsvergleich zwischen Österreich und Deutschland, noch unveröffentlichte Dissertation, Wien

Höland, A./Kahl, U. /Zeibig, N. (2007): Kündigungspraxis und Kündigungsschutz im Arbeitsverhältnis. Eine empirische Praxisuntersuchung aus Sicht des arbeitsgerichtlichen Verfahrens. Schriften der Hans-Böckler-Stiftung, Baden-Baden

Krause, A. (2006a): Die Einstellung zum Kündigungsschutz - wie wichtig sind Gerechtigkeitsnormen und Entlassungserfahrungen?, in: Struck, O./Stephan, G./Köhler, C./Krause, A./Pfeifer, C./Sohr, T.: Arbeit und Gerechtigkeit. Entlassungen und Lohnkürzungen im Urteil der Bevölkerung, Wiesbaden, S. 105-119

Krause, A. (2006b): Labour market reforms in the light of a new justice principle: from redistribution to equal access, MS, Budapest, http://www. ceu.hu/polsci/brownbag_papers/0607/alexandra_krause.pdf

Krause, A./Pfeifer, C./Sohr, T. (2006): Was beeinflusst die Akzeptanz von Entlassungen und Lohnkürzungen, in: Struck, O./Stephan, G./Köhler, C./Krause, A./Pfeifer, C./Sohr, T.: Arbeit und Gerechtigkeit. Entlassungen und Lohnkürzungen im Urteil der Bevölkerung, Wiesbaden, S. 33-69

Leuthner, R./Achitz, B./Farny, O./Wöss, J. (2003): Abfertigung neu - Betriebliches Mitarbeitervorsorgegesetz, Gesetze und Kommentare NR 179, Wien

Leventhal, G. S. (1980): What should be done with equity theory? New approaches to the study of fairness in social relationship: in Gergen, K. J./ Greenberg, M. S./Willis, R. H. (eds.): Social exchange. Advances in theory and research, New York, London, pp. 27-55

Lind, E. A./Tyler, T. R. (1988): The Social Psychology of Procedural Justice, New York
Luhmann, N. (1983): Legitimation durch Verfahren, Frankfurt am Main (stw 443)

Machura, S. (1993): N. Luhmanns "Legitimation durch Verfahren“ im Spiegel der Kritik, Zeitschrift für Rechtssoziologie 14, S. 97-114 Machura, S. (2000): Fairness und Legitimität, Baden-Baden Machura, S. (2003): Ehrenamtliche Richter in Südrussland. Eine empirische Untersuchung zu Fairness und Legitimität, Münster Machura, S. (2006): Ehrenamtliche Verwaltungsrichter, Münster Magen, S. (2005a): Fairness, Eigennutz und die Rolle des Rechts. Eine Analyse auf Grundlage der Verhaltensökonomie. Reprints of the Max Planck Institute for Research on Collective Goods 22, Bonn Magen, S. (2005b): Zu einer Verhaltenstheorie der Gerechtigkeit, Vortrag anlässlich der Eröffnungsfeier des Max-Planck-Instituts zur Erforschung von Gemeinschaftsgütern, Bonn, http://www.coll.mpg.de/pdf_dat/ Magen\%20Verhaltenstheorie\%20der\%20Gerechtigkeit.pdf Müller-Glöge, R. (2006): in: Dieterich, T./Hanau, P./Schaub, G. (Hrsg.), Erfurter Kommentar zum Arbeitsrecht, München.

Pfarr, H./Ullmann, K./Bradtke, M./Schneider, J./Kimmich, M./

Bothfeld, S. (2005): Der Kündigungsschutz zwischen Wahrnehmung und Wirklichkeit, München und Mering

Pfeifer, C. (2004): Fairness und Kündigungen. Eine theoretische und empirische Analyse, in: Zeitschrift für Arbeitsmarktforschung 2, S. 127-145 Rawls, J. (1993): Eine Theorie der Gerechtigkeit, Frankfurt am Main (stw 271)

Röhl, K. F. (1987): Rechtssoziologie. Ein Lehrbuch, Köln, Berlin u. a. Röhl, K. F. (1993): Verfahrensgerechtigkeit (Procedural Justice). Einführung in den Themenbereich und Überblick, in: Zeitschrift für Rechtssoziologie 14, S. 1-34

Röhl, K. F. (ed.) (1997): Procedural Justice, Oñati international series in law and society, Aldershot u. a.

Rudolph, K. (1983): Der Vergleich im Zivilprozess aus Sicht des Richters, in: Gottwald, W./Hutmacher, W./Röhl, K. F./Strempel, D.: Der Prozessvergleich, Köln, S. 35-38

Thibaut, J./Walker, L. (1975): Procedual Justice. A Psychological Analysis New Jersey

Tyler, T. R. (1990): Why People Obey the Law, New Haven u. a.

Tyler, T. R. (ed.) (2005): Procedural Justice, vol. 1 und 2, Aldershot et al. Sadowski, D. (2002): Personalökonomie und Arbeitspolitik, Stuttgart Stephan, G. (2006): Fair geht vor. Was die Leute von Entlassungen und Lohnkürzungen halten, IABKurzbericht 1

Stephan, G./Struck, O./Köhler, C. (2006): Warum eine Studie zum Thema "Arbeit und Gerechtigkeit"?, in: Struck, O./Stephan, G./Köhler, C./Krause, A./Pfeifer, C./Sohr, T.: Arbeit und Gerechtigkeit. Entlassungen und Lohnkürzungen im Urteil der Bevölkerung, Wiesbaden, S. 11-16 Struck, O./Stephan, G./Köhler, C./Krause, A./Pfeifer, C./Sohr, T. (2006): Arbeit und Gerechtigkeit. Entlassungen und Lohnkürzungen im Urteil der Bevölkerung, Wiesbaden

Struck, O. (2006): Arbeitsmotivation, Fluktuation, Krankenstand - wie wirken sich Entlassungen und Lohnkürzungen aus?, in: Struck, O./ Stephan, G./Köhler, C./Krause, A./Pfeifer, C./Sohr, T.: Arbeit und Gerechtigkeit. Entlassungen und Lohnkürzungen im Urteil der Bevölkerung, Wiesbaden, S. 87-104

Wemmers, J. (1996): Social Psychological Explanations of the „Fair Process Effect “. A Testing of the Group Value Model, in: Zeitschrift für Rechtssoziologie 17, S. 114-134 